\title{
Sindrome de estrés laboral asistencial en docentes de la UNAN-Managua, FAREM- Carazo, en el segundo semestre 2019
}

\author{
Br. Bismarck Santiago Mena Aburto \\ Estudiante de Licenciatura en Psicología \\ UNAN-Managua, FAREM-Carazo \\ bicksantiago25@gmail.com

\section{Br. Danny Raphael Cruz Alemán} \\ Estudiante de Licenciatura en Psicología \\ UNAN-Managua, FAREM-Carazo \\ drca242@gmail.com
}

\section{Br. Angelie Michelle Guevara García \\ Estudiante de Licenciatura en Psicología \\ UNAN-Managua, FAREM-Carazo \\ angelie1996@gmail.com}

Fecha de recepción: 28 de enero, 2020 / Fecha de aceptación: 23 de marzo, 2020

https://doi.org/10.5377/torreon.v9i25.9852

Palabras clave: estrés, docentes, síndrome de estrés laboral asistencial, prevalencia.

\section{RESUMEN}

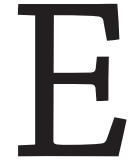

1 estrés es la caracterización de signos y síntomas que afectan a niveles físicos y psicológicos a las personas. Es común en los ámbitos laborales, en específicamente en aquellos donde se prestan servicios o asistencia a terceros, esto es denominado como "síndrome de estrés laboral asistencial".

El presente estudio indaga sobre este síndrome en los docentes que integran los departamentos académicos de la UNAN-Managua; FAREM-Carazo, durante el segundo semestre 
del 2019 algunas conductas o comentarios de los docentes hacia los estudiantes se consideraron como indicadores de la manifestación del síndrome de estrés laboral asistencial, estas califican dentro de la teoría de Jackson \& Maslach sobre el problema de estudio por tanto se determinó evaluar la prevalencia de este síndrome con el fin de indicar la relación existente entre las variables sociodemográficas de sexo y edad como factores que requieren de atención especial, para ello, se realizó un estudio de tipo descriptivo, de corte transversal característico de estudios cuantitativos.

El estudio incluye un análisis teórico relacionado al problema en estudio, así mismo, se utilizó un instrumento estandarizado que mide las diferentes dimensiones donde el estrés se manifiesta en un individuo. Dicho instrumento psicométrico permitió recolectar datos estadísticos que fueron procesados en los programas estadísticos correspondientes utilizando estadística descriptiva, estos resultados fueron analizados desde los enfoques teóricos abordados permitiendo hacer una discusión sobre la prevalencia del estrés laboral asistencial concluyendo que existe una baja presencia de este síndrome en los docentes de la FAREM, Carazo.

\section{INTRODUCCIÓN}

El presente artículo tiene como objetivo principal evaluar el estrés laboral asistencial en docentes de la UNAN Managua, FAREM Carazo, en el segundo semestre del año 2019, para ello se debe determinar la prevalencia existente de estrés laboral asistencial de los docentes, con el fin de describir los niveles de cansancio emocional, despersonalización y realización personal e indicar la prevalencia de este síndrome según el sexo y la edad. Se entiende por estrés, como las respuestas psicológicas y fisiológicas ante un evento o situación que afecta el bienestar de una persona, según la APA (2014) el estrés es un "patrón de respuestas específicas y no específicas que una persona experimenta ante los estímulos que perturban su equilibrio y que retan o exceden su capacidad de hacerles frente" p. 822.

El estrés laboral se genera a partir de las demandas que existen en una organización y del ambiente del mismo, esto ha sido el punto de partida de este estudio, puesto que el abordaje del tema en estudios anteriores se ha limitado a evaluaciones de desempeño laboral en organizaciones que no describen precisamente el estrés laboral asistencial. Los autores García R., Gelpi M., Cano V., \& Catalina R. (2009) definen el estrés laboral asistencial como "un estado de tensión física y psicológica que se produce cuando existe un desequilibrio entre las demandas que plantean la organización del trabajo y el medio laboral, y los recursos de los trabajadores para hacerles frente" (p. 44).

El desgaste laboral y ocupaciones propician la aparición del estado expuesto en los docentes, ante todo es necesario evaluar ¿Cuál es la prevalencia de estrés laboral asistencial al 
que están expuestos los docentes de la FAREM-Carazo, en el segundo semestre 2019? Con el fin de describir y contextualizar nuevos datos en relación al tema, así mismo, por la metodología implementada se permitirá establecer la prevalencia o no del síndrome de estrés laboral asistencial permitiendo el desarrollo de nuevos estudios y la creación de nuevas hipótesis relacionadas al caso, así como brindar un aporte académico sustentado en los resultados obtenidos, analizados y discutidos.

\section{MÉTODOS}

\section{Tipo de estudio}

El estudio es una investigación aplicada, se empleó el enfoque cuantitativo, el cual permitió determinar, describir e indicar el estrés laboral asistencial en docentes de la UNANManagua, FAREM-Carazo; se utilizó un diseño no experimental de corte transversal, con un alcance descriptivo.

\section{Participantes}

La muestra está compuesta por 99 docentes de la UNAN-Managua, FAREM-Carazo, la cual se obtuvo por medio del programa STATS ${ }^{\mathrm{TM}}$ 2.0. Esta muestra se divide en tres departamentos; 45 docentes del Departamento de Ciencias de la Educación y Humanidades, 28 docentes del Departamento de Ciencias, Tecnología y Salud, 26 docentes del Departamento de Ciencias Económicas y Administrativas, esta muestra fue seleccionada por medio de la fórmula para la obtención de subgrupos de muestras:

$$
k h=\frac{n h}{N H}
$$

Ante la negativa de algunos docentes de participar en este estudio, pese a la anuencia y autorización solicitada previamente a cada uno de los directores delos departamentos académicos, y el subsidio laboral en el que algunos docentes se encontraban en el momento de la aplicación, impidió que se aplicaran los instrumentos a su totalidad por lo que únicamente se aplicó 73 instrumentos (37 docentes del Departamento de Ciencias de la Educación y Humanidades, 19 docentes del Departamento de Ciencias de Económicas y Administrativas y 17 docentes del Departamento de Ciencias, Tecnología y Salud, el margen de error varió al 7\% basándose en la fórmula para estudios sociales, este margen de error es aceptable para estudios de índole social:

$$
d^{2}=Z^{2} p \cdot q \frac{(N-n)}{n} / n(N-1)
$$


El muestreo del estudio es de tipo probabilístico estratificado, el cual consiste en la división de la población en diferentes subgrupos o estratos, dichos estratos son los departamentos académicos de la FAREM-Carazo. La elección de este tipo de muestreo se realizó con la intención de obtener resultados equitativos mediante la evaluación de muestras más precisas.

\section{INSTRUMENTOS}

Se aplicó el Inventario Burnout de Maslach (MBI, por sus siglas en inglés), la cual consta de tres dimensiones que se evalúan individualmente, las cuales se procesaron en el programa Microsoft Excel versión 2013 para la realización de una base de datos utilizada para introducir en una segunda etapa en el programa estadístico SPSS versión 18 y Microsoft Excel 2013 los datos obtenidos, con el fin de obtener los resultados estadísticos de la investigación.

\section{RESULTADOS}

La definición de estrés como tal, es adaptada al contexto, dependiendo de las literaturas y las teorías, la designación de estrés descrita en este estudio corresponde a la que presentan personas que tiene trabajos de asistencias como la docencia, servicios de salud y seguridad.

Maslach \& Jackson (1997), Postulan tres dimensiones del estrés, estas abordan las áreas psicológicas, motivacionales y personales del individuo, al clasificarlas dan una pauta para determinar los niveles de estrés que existen en la persona.

Sustancialmente cabe aclarar que el MBI y sus dimensiones de evaluación no miden niveles de estrés presente en una población, sino, busca indicar la prevalencia mediante la correlación de las dimensiones que lo componen.

En el gráfico 1 se representa el estrés laboral asistencial presente en los docentes de la FAREM Carazo, este resultado se obtuvo en la corrección individual de las dimensiones del estrés laboral asistencial en donde dos de los evaluados que corresponde al $2.7 \%$ alcanzaron puntajes que indican la presencia de estrés laboral asistencial, mientras el 97.3\% refleja que los docentes no poseen o indican la presencia de este sino más bien a factores externos que generan otros tipos de estrés que no están ligados al ámbito de la docencia. 


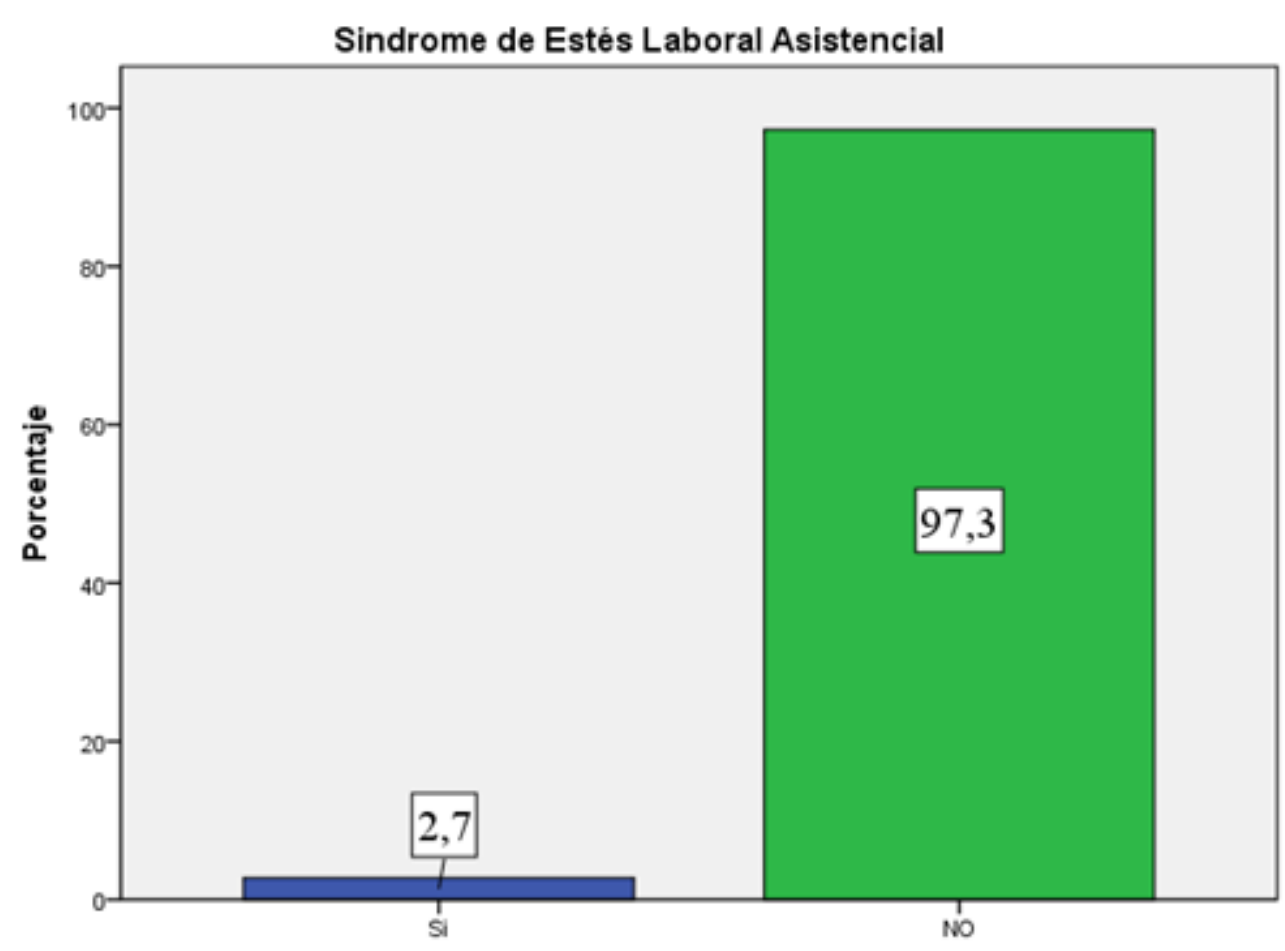

Gráfica 1. Síndrome de estrés laboral asistencial

En el gráfico 2 se representan la dimensión de cansancio emocional dando por resultado que el $8.2 \%$ tiene Cansancio emocional, mientras que el $24.7 \%$ presenta un cansancio medio, y un $67.1 \%$ restante proyecta un cansancio bajo.

$\mathrm{Al}$ obtener una puntuación alta en esta dimensión, significa que la persona se encuentra en situaciones en las cuales se siente emocionalmente exhausto provocado por su entorno laboral.

Estas situaciones podrían provocar repercusiones en el estado de ánimo del individuo como sentimientos de apatía, melancolía e inutilidad y tener derivaciones en el área social y conductual, sin embargo, a nivel global en esta dimensión predomina el indicador de cansancio emocional bajo, exponiendo que los sujetos evaluados con las puntuaciones bajas manifiestan sentimientos de agrado y satisfacción en su entorno laboral. 


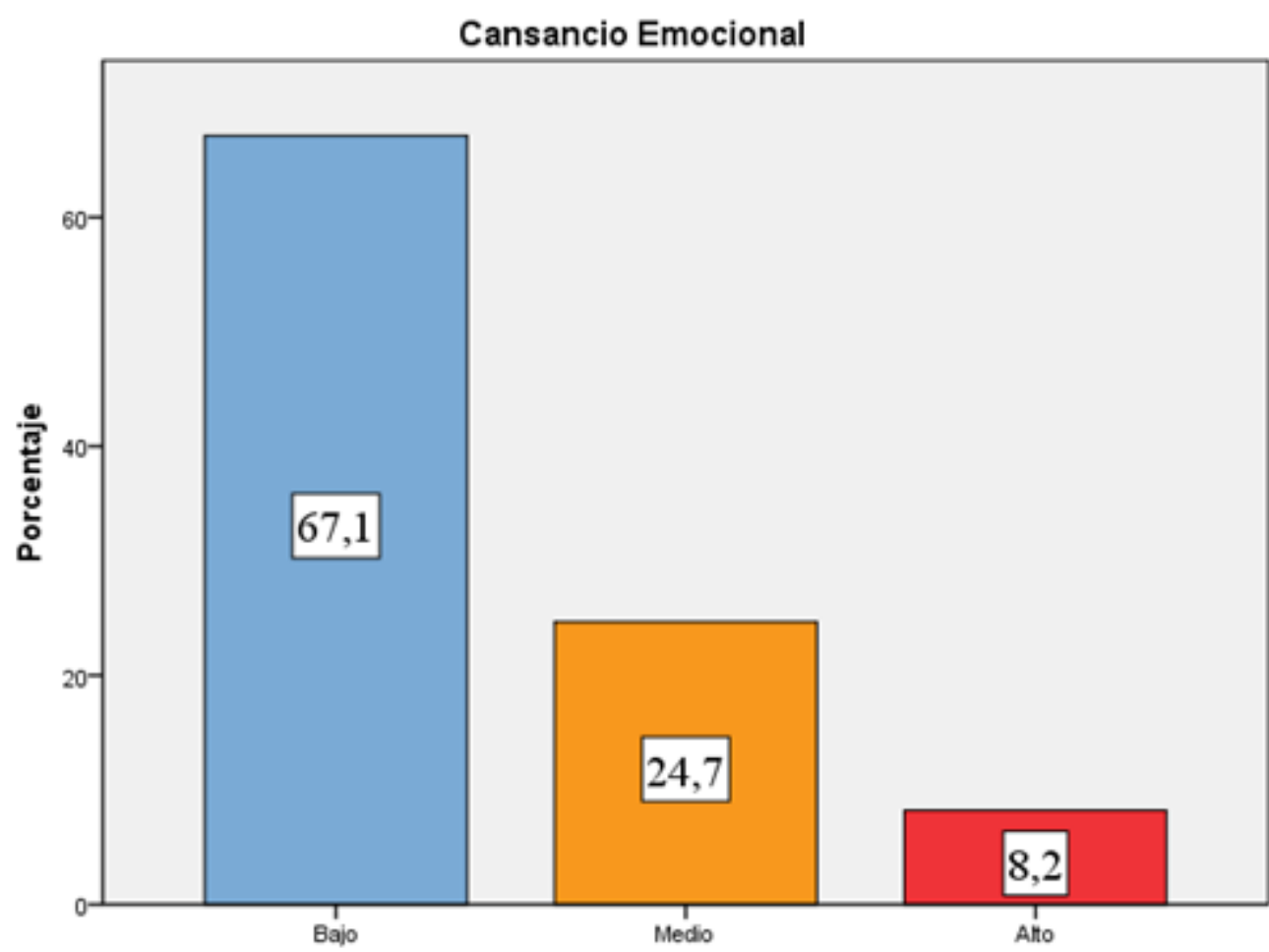

Gráfica 2. Cansancio emocional

En la gráfica 3 corresponde a la dimensión Despersonalización en la cual la muestra evaluada dio como resultado que el $13.7 \%$ de los docentes poseen baja despersonalización, mientras que el $38.4 \%$ muestra una despersonalización media y el $47.9 \%$ restante de la muestra proyecta una despersonalización alto.

La dimensión de Despersonalización se entiende como las respuestas que manifiesta el individuo evaluado sobre los elementos o situaciones que experimenta en su entorno laboral, si la puntuación es alta en esta dimensión, expone que el individuo expresa respuestas frías o actúa como un observador externo ante eventualidades cotidianas de sus funciones dirigida a los receptores o a los cargos que ostenta el profesional.

En esta dimensión predomina la puntuación alta, lo cual refiere que los docentes evaluados actúan ante ciertas situaciones en dependencia de su personalidad con apatía, negatividad y cinismo mientras que otros tratan al menos de lidiar esas situaciones brindando respuestas que no les agradan o bien con una actitud neutra. 


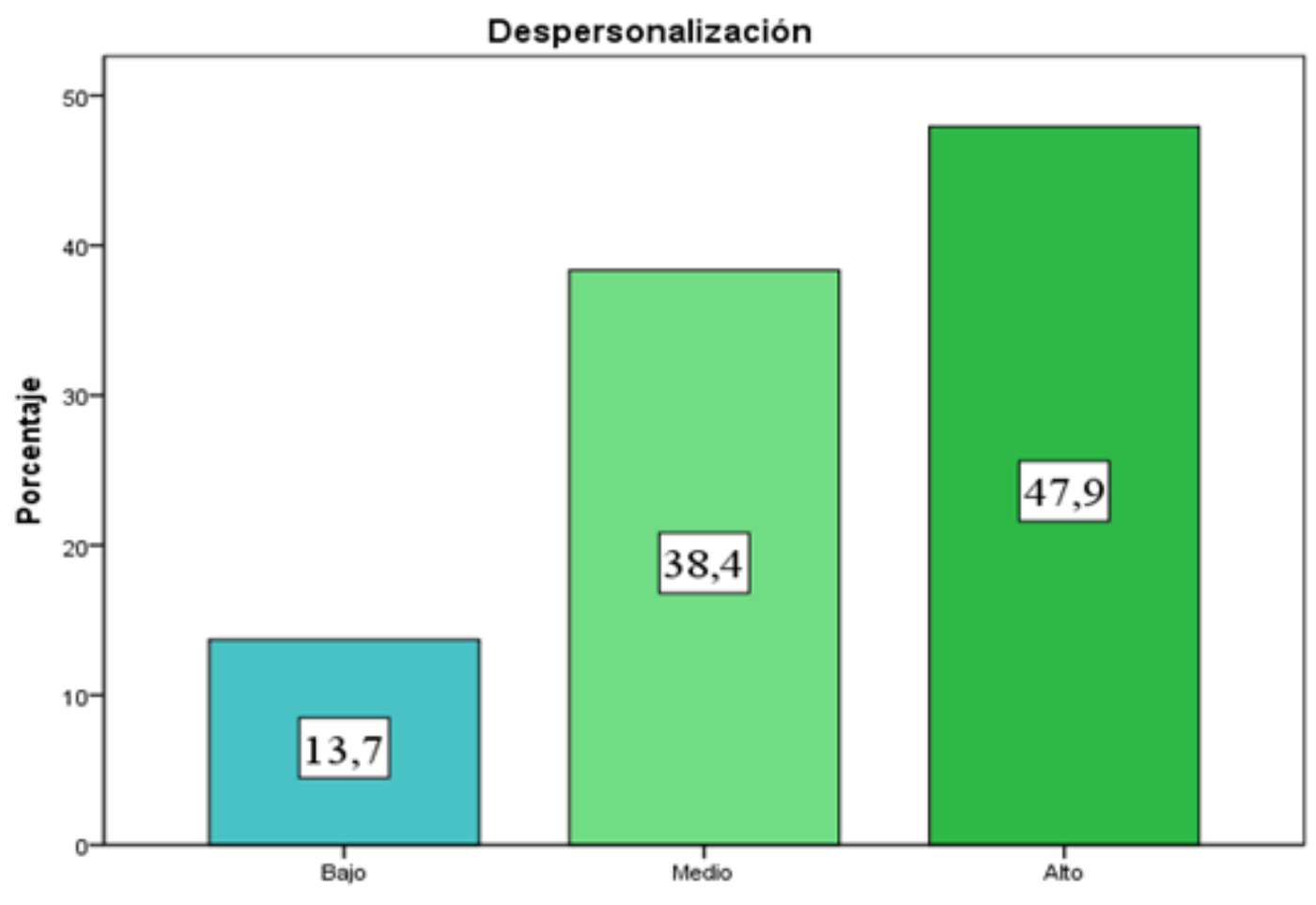

Gráfica 3. Despersonalización

En la gráfica 4 se representa la realización personal que poseen los docentes evaluados, los resultados obtenidos exponen que el $67.1 \%$ de la muestra tiene una baja realización personal, el 24.7 \% de la muestra evaluada posee una Realización Personal media y el 8.2\% de la muestra presenta una alta realización con respecto con el puesto que desempeña.

En la dimensión de Realización Personal una puntuación baja es muestra de que el profesional tiene una perspectiva de bajo rendimiento o de poca competencia en el desarrollo de su trabajo, manifestando sentimientos de agotamiento, desagrado, decepción o baja autoestima, que sus expectativas han sido desplazadas y sus funciones no las realza por competencia, si no como una obligación de su labor.

Sólo un 8.2 \% de los evaluados puntuaron una alta realización personal, lo cual equivale a que tiene una alta autoestima, sintiéndose a gusto en la posición en la que se encuentra en su centro de trabajo y en el desempeño de sus labores contrario al $61.7 \%$ que refieren que sus labores son una carga que no ha sustentado sus necesidades personales y académicas. 


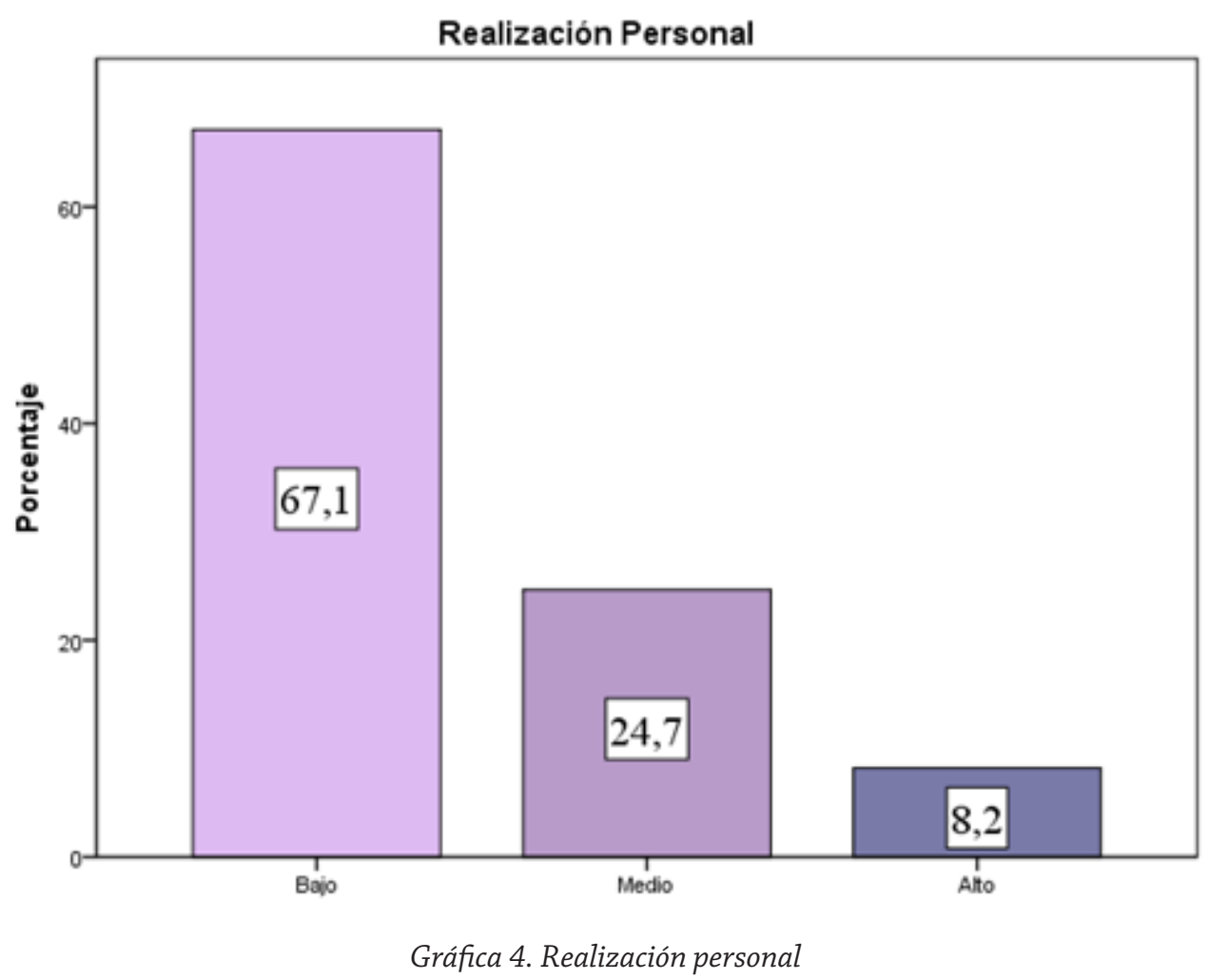

En el gráfico 5 se muestra la prevalencia del estrés laboral asistencial en docentes en función del sexo, se observa que el 3\% de la muestra perteneciente al sexo femenino y el 3\% que pertenece al sexo masculino, padecen de estrés laboral asistencial, contrario a ello, el $97 \%$ de la muestra de ambos sexos, no presenta esta condición.

Este resultado indica que la prevalencia el sexo y el estrés laboral asistencial afecta por igual a los docentes del sexo masculino como del femenino, al evaluar a la muestra existe la tendencia que el síndrome de estrés laboral asistencial afecta por igual a hombres y a mujeres de esta institución. 


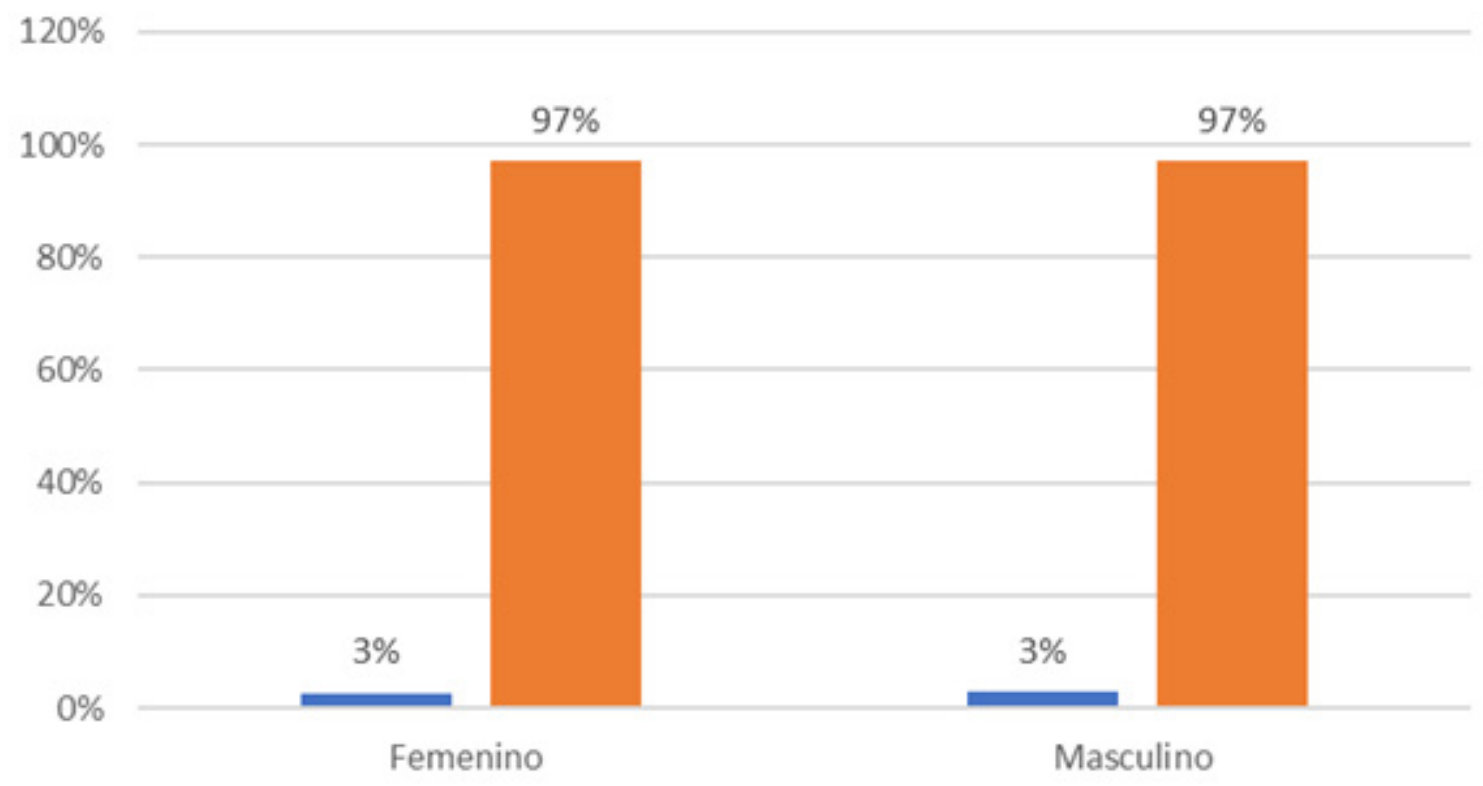

\section{$\square \mathrm{SI}=\mathrm{NO}$}

Gráfica 5. Prevalencia del estrés laboral asistencial en docentes

En el 6 gráfico se muestra la prevalencia del estrés laboral asistencial en función de la edad, de la muestra evaluada, en la cual se observa que el $100 \%$ de los evaluados que comprenden las edades entre 29-39 años no padecen de esta condición,

El $94.45 \%$ que comprenden las edades entre $40-59$ años, no presentan de esta circunstancia, pero en este mismo rango de edad existe un $5.55 \%$ que sí presentan este estado, cabe señalar que un $100 \%$ de los evaluados que no indican su edad, tampoco manifiestan que sufran del estrés laboral asistencial. 


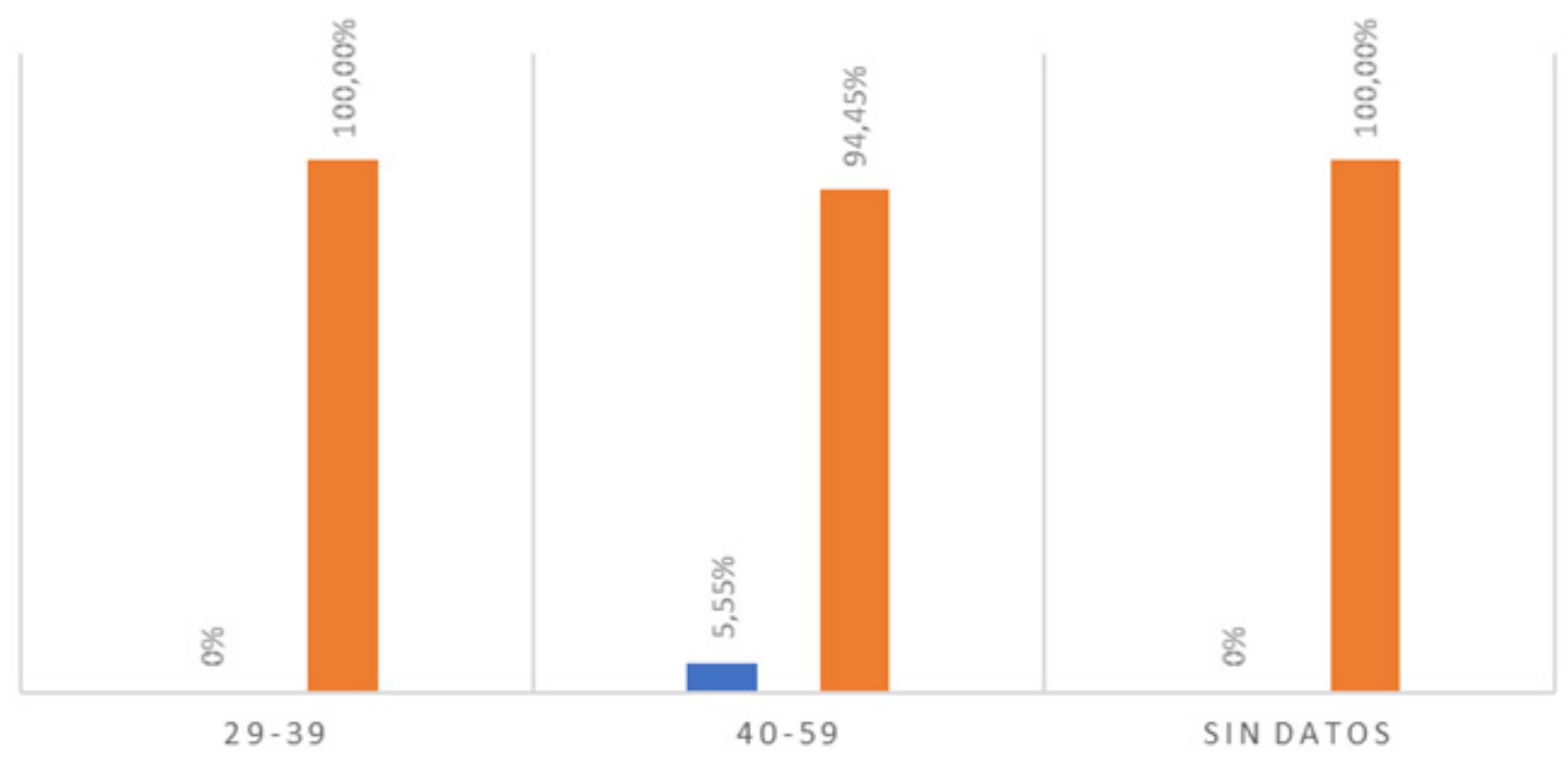

Gráfica 6. Prevalencia del estrés laboral asistencial en función de la edad

\section{DISCUSIÓN}

El estrés es una de respuestas que el cuerpo emite precipitadamente ante un estímulo no grato o esperado, estas respuestas podrían conllevar reacciones a nivel cognitivo, afectivo o conductual a grandes rasgos dependiendo de cómo la persona está preparada para asumir tales situaciones. La APA (2013) define al estrés como un "patrón de respuestas específicas y no específicas que una persona experimenta ante los estímulos que perturban su equilibrio y que retan o exceden su capacidad de hacerles frente” p. 822. Esta definición también vincula al estrés hacia las alteraciones que puede interferir en el bienestar y en el equilibrio de una persona afectando de manera considerable la vida cotidiana de la persona.

Según los planteamientos de las referencias de este estudio, expresa que las principales manifestaciones que podría tener una persona que padece de estrés están a nivel cognitivo, afectivo y conductual, las cuales se manifiestan en múltiples formas como en las reacciones que podría tener la persona a situaciones; a raíz de un episodio de estrés como la agresividad, resignación, pensamientos de inutilidad o insatisfacción, podría embargar la mente de una persona junto con sentimientos de ira, tristeza y apatía, afectando su relación con su entorno social, laboral y familiar (Betancur, 2016).

Por medio de la aplicación del Inventario Burnout Maslach (MBI), un instrumento que indica la prevalencia del síndrome en estudio, se encontró que la presencia de estrés laboral 
asistencial es baja en el cuerpo de docentes, porque solamente 2 docentes tienen en consonancia las tres dimensiones requeridas para confirmar la presencia del síndrome, por lo que se descarta el planteamiento realizado en la hipótesis 1 dónde se indicaba que la prevalencia del estrés laboral asistencial en los docentes de la FAREM-Carazo, es mayor al 50\%, el resultado obtenido es por debajo de este planteamiento.

Los docentes que conforman este centro de educación superior, es un grupo susceptible de sufrir de estrés por el ámbito laboral en el que se desenvuelven, al ser docentes universitarios siempre están en constante interacción con terceros (alumnos) los cuales tienen diversas formas de pensar y comportarse con diferentes rangos de edades y condiciones psicosociales que podrían interferir en las relaciones y el ambiente dentro del aula de clases.

El Inventario Burnout Maslach (MBI) está conformado por tres dimensiones en las cuales se complementan para determinar si la persona dentro de la muestra evaluada presenta estrés.

En la primera dimensión que corresponder a cansancio emocional, dio como resultado un nivel bajo descartando la hipótesis 2 de este estudio, al ser contrario el resultado al planteamiento realizado.

Los docentes con este nivel de cansancio emocional bajo, presentan en su mayoría, sentimientos de agrado y satisfacción, naturalmente algunos de los docentes presentan un grado medio de agotamiento, mientras que algunos casos aislados, presentan un nivel de cansancio emocional alto lo que nos demuestra que están emocionalmente agotados, y podrían manifestar apatía, melancolía o inutilidad.

En cuanto a la Despersonalización, dio como resultado que una mayoría de los docentes presentan un alto nivel, lo que significa que en el área laboral sus acciones son de apatía, cinismo, suelen dar respuestas frías y se ven como un agente externo en su entorno académico. Las valoraciones estadísticas confirman que aunque los docentes no sufran de estrés laboral asistencial, pueden reaccionar ante los estímulos del ambiente laboral con respuestas o acciones que se consideran anómalas e inaceptables para la convivencia, evidencia de ello fue el trato de una buena parte de los docentes hacia los evaluadores, algunos fueron inaccesibles mientras otros discriminaron negativamente con comentarios o gestos faciales de displacer, confirmando los planteamientos teóricos de Maslach y Jackson y el realizado en la hipótesis 3 de este estudio.

La última dimensión que posee el test es Realización Personal, comprende como el docente se siente en la posición en la que se encuentra en su centro de labor, el resultado obtenido indica un nivel bajo de realización lo que confirma el planteamiento de la 4 hipótesis, muestra de que tienen la percepción de tener un bajo rendimiento y tener grados de decepción, agotamiento o desagrado con respecto a su posición y labor. 
El resultado anterior, complementa los datos de altos niveles de despersonalización, sin embargo, el instrumento requiere que el cansancio emocional y la despersonalización estén en consonancia para indicar que existe prevalencia de estrés laboral asistencial. En esta investigación la consonancia discrepa, el nivel de realización personal y los niveles de cansancio emocional son similares indicando que paralelamente los que menos se esfuerzan tendrán menos realización personal.

Estos resultados no se pueden contrastar con los estudios de referencia a este debido a que se han encontrado errores en la corrección de este instrumento en las investigaciones anteriores debido a que los evaluadores desconocen los procedimientos paramétricos de un instrumento psicológico de tipo paramétrico, sin embargo, el planteamiento de la teoría de Maslach y Jackson se confirma y respalda teóricamente los resultados del fenómeno en cuestión.

La prevalencia de estrés laboral asistencial en relación a los géneros, indica que existe 1 caso por cada género, es decir, la prevalencia entre varones y mujeres de estrés laboral asistencial será del 1\% para los docentes del sexo masculino y femenino, descartando la hipótesis 5, debido a que según este estudio el estrés afecta a hombres y a mujeres por igual, así mismo, la relación del síndrome de estrés laboral asistencial y la edad demuestra que entre más años cumplidos habrá más tendencia a estar expuestos al estrés, confirmando lo planteado por Betancurt en su estudio de 2016 que expresa que todos los sujetos están expuestos al estrés sin importar el sexo y la edad.

Los presentes resultados no difieren de una realidad académica en donde los docentes ejercen sus funciones como una obligación y no una vocación, los niveles de realización personal son considerablemente bajos puesto que algunos docentes indican que no han alcanzado sus metas y objetivos personales en relación a su oficio.

En la caracterización de un proceso de evaluación de rendimiento organizacional pueden cumplirse las metas y objetivos de la institución, pero el personal no está respondiendo a sus expectativas, es donde la frustración, la apatía y el trato despersonalizado o minimizado hacia terceros provocan la necesidad de deshacerse de tareas que les resultan estresantes y que no tienen relación directa con las funciones de docente.

En términos generales, el tipo de estrés que se está manifestando en esta institución es el estrés denominado "Eustrés", un estrés de tipo positivo que se presenta al realizar acciones en búsqueda del cumplimiento de los objetivos organizacionales, los cuales si tienen que ver con el quehacer académico, pero hay que considerar que el cumplimento de los objetivos que se plantea la institución está llevando a una buena parte de los docentes a no cumplir sus metas personales y es ahí donde dos de los docentes evaluados ya han presentado indicadores de sufrir estrés laboral asistencial. 


\section{CONCLUSIONES}

- La prevalencia de estrés laboral asistencial de los docentes de la UNAN-Managua; FAREM Carazo es baja pero los resultados de las dimensiones indican que existen afectaciones que podrían contribuir a la aparición de estrés, aunque no corresponde precisamente al síndrome en estudio.

- Según los datos obtenidos el nivel de cansancio emocional es predominantemente bajo, es decir, los docentes presentan un bajo desgaste emocional respecto al ejercicio de sus funciones y que en ellas alcanzan cierto grado de compensación emocional.

- Se establece que la despersonalización en los docentes de la FAREM Carazo es alto, presentan apatía, respuestas impersonales, frialdad, respuestas desinteresadas, el resultado es evidente durante el proceso de recolección de datos debido a la actitud que algunos de los evaluados fueron desafiantes, negativo y poco interesada.

- Según los resultados obtenidos el nivel de realización personal es bajo, el problema encontrado plantea que los docentes no se sienten conformes con su ejercicio profesional dado que sus expectativas laborales no cumplen las personales, aunque ellos están desempeñando plenamente sus funciones profesionales, ellos no están cumpliendo sus expectativas personales.

- Se indica que la relación entre el estrés laboral asistencial en los docentes de la FAREM Carazo y el sexo no es significativa, no se puede afirmar la teoría establecida que indica que el estrés en estudio afecta en su mayoría a las mujeres y que este puede presentarse en ambos géneros.

- Se indica que la presencia de estrés laboral asistencial en docentes de la FAREM-Carazo es más evidente en sujetos de mayor edad, es decir a más edad mayor probabilidad de sufrir estrés laboral asistencial.

\section{REFERENCIAS BIBLIOGRÁFICAS}

Aamodt, M. G. (2010). Psicología industrial/ organizacional 6a. edición. México D.F: Cengage Learning.

Asociación Americana de Psiquiatría, Manual diagnóstico y estadístico de los trastornos mentales $\left(\mathrm{DSM}-5^{\circledR}\right)$, 5a Ed. Arlington, VA, Asociación Americana de Psiquiatría, 2014.
APA. (2010). Centro de apoyo de la A.P.A. Disponible el 11 de septiembre de 2019, del Centro de Apoyo de la Asociación de Psicología Americana. Obtenido de https://www.apa.org/centrodeapoyo/ tipos 
Barraza Macías, A., \& Jaik Dipp, A. (2011). Estrés, burnout y bienestar subjetivo, Investigaciones sobre la salud mental de los agentes educativos. Durango: Instituto Universitario Anglo Español.

Betancur, M. (2016). El estrés laboral en los empleados de la Facultad De Ciencias Agrarias De La Universidad De Antioquia. Medición y mecanismos de afrontamiento. Obtenido de Repositorio digital FCSH: http://200.24.17.74:8080/ jspui/handle/fcsh/523

Chiavetano, I. (2009). Comportamiento organizacional, La dinámica del éxito en las organizaciones; Segunda edición. México D.F.: McGraw-Hill/Interamericana Editores, S.A. de C.V.

El-Sahili, L. (2010). Psicología para el Docente. Consideraciones sobre los riesgos y desafíos de la práctica magisterial. Guadajara, México.: Universidad de Guanajuato.

García, J., Gelp, J., Cano, A., \& Catalina, C. (2009). Manual Ibermutuamur. Cómo combatir el estrés laboral. Madrid: Editorial y Producción Arteanima, S.L.

Gonzales, L. F. (2010). Psicología para el Docente: Consideraciones sobre los riesgos y desafíos de la práctica magisterial. Guadajara: Universidad de Guanajuato.

Herranz, J., Reig-Ferrer, A., \& Cabrero, J. (2006). La prevalencia del estrés laboral asistencial entre los profesores universitarios. Obtenido de RUA. Repositorio Institucional de la Universidad de Alicante: http://hdl. handle.net/10045/25008

Lazarus, R. (2000). Estrés y emoción manejo e implicaciones en nuestra salud. Madrid, España.: Editorial Desclée De Brouwer.

Marulanda Ruiz, I. C. (2009). Estrés laboral enemigo silencioso de la salud mental y la satisfacción con la vida, segunda edición. Bogotá, Colombia: Ediciones Uniandes.

Maslach, C., \& Jackson, S. (1997). Inventario Burnout de Maslach. Madrid, España.: TEA Ediciones.

Pérez, P. (2015). Descripción estado de Burnout, antes y después de un programa de entrenamiento psicológico, mediante la escala MBI, en un grupo de docentes del colegio Gimnasio Pilares del Saber del municipio de Turbo. Obtenido de Repositorio digital FCSH: http://200.24.17.74:8080/jspui/handle/ fcsh/337

Sampieri, R. (2014). Metodología de la Investigación Sexta Edición. México D.F., México.: McGraw-Hill Education.

Sarason, I., \& Sarason, B. (2006). Psicopatología, Psicología anormal. El problema de la conducta inadaptada. México D.F., México.: Pearson Education.

Torres, X., \& Bailles, E. (2015). Comprender el estrés. Barcelona, España: Amat Editorial. 